Published in Surface Topography: Metrology and Properties, 2021, vol. 9, article no. 025021, which should be cited to refer to this work. DOI:http://dx.doi.org/10.1088/2051-672X/abfa28

\title{
Extension and validation of a revised Cassie-Baxter model for tailor-made surface topography design and controlled wettability
}

Nikolaos Lempesis, ${ }^{1,2, *}$ Rudolf J. Koopmans, ${ }^{1,2}$ Ruth Díez-Ahedo ${ }^{3}$, Per Magnus Kristiansen ${ }^{4,5,6}$

${ }^{1}$ Plastics Innovation Competence Center, Passage du Cardinal 1, CH-1700 Fribourg, Switzerland

${ }^{2}$ Department of Mechanical Engineering, College of Engineering and Architecture Fribourg HES-SO, Bd de Pérolles 80, CH-1705 Fribourg, Switzerland

${ }^{3}$ Tekniker, Iñaki Goenaga 5, 20600 Eibar, Spain

${ }^{4}$ FHNW University of Applied Sciences and Arts Northwestern Switzerland, School of Engineering, Institute of Polymer Nanotechnology (INKA), Klosterzelgstrasse 2, CH-5210 Windisch, Switzerland

${ }^{5}$ Laboratory for Micro- and Nanotechnology, Paul Scherrer Institute, 5232 Villigen PSI, Switzerland

${ }^{6}$ University College Dublin, School of Mechanical and Material Engineering, Belfield, Dublin 4, Ireland

\section{${ }^{*}$ Author to whom correspondence should be addressed: nikolaos.lempesis@hefr.ch}

Keywords: wettability, wetting model, topography, Cassie-Baxter, hydrophobic, omniphobic

\begin{abstract}
Predicting wettability accurately across various materials, surface topographies and wetting liquids is undeniably of paramount importance as it sets the foundations for technological developments related to improved life quality, energy saving and economization of resources, thereby reducing the environmental impact for recycling and reuse. In this work, we extend and validate our recently published wetting model, constituting a refinement of the original Cassie-Baxter model after consideration of realistic curved liquid-air interfaces. Our model enabled more meaningful contact angle predictions, while it captured the experimentally observed trends between contact angle and surface roughness. Here, the formalism of our
\end{abstract}


wetting model is further extended to 3D surface topographies, whereas the validity of our model, in its entirety, is evaluated. To this end, a total of thirty-two experimentally engineered surfaces of various materials exhibiting single- and multilevel hierarchical topographies of increasing complexity were utilized. Our model predictions were consistently in remarkable agreement with experimental data (deviations of 3-6\%) and, in most cases, within statistical inaccuracies of the experimental measurements. Direct comparison between experiments and modeling results corroborated that surface topographies featuring re-entrant geometries promoted enhanced liquid-repellency, whereas hierarchical multilevel surface topographies enabled even more pronounced nonwetting behaviors. For the sinusoidal topography, consideration of a second superimposing topography level almost doubled the observed water contact angles, whereas addition of a third level brought about an extra $12.5 \%$ increase in water contact angle.

\section{Introduction}

Everyday life commodities, such as food, water and other beverages, as well as inedible domestic products, such as cosmetics and liquid detergents are stored, transported and maintained within plastic or glassy containers. Adhesion of these goods to their packaging material is responsible for product waste [1], poor product appearance [2], increased package recycling costs [3], as well as sanitary problems and production deterioration in industrial units due to fouling of equipment [4]. Wettability was identified as the most dominant mechanism related to adhesion of a liquid onto a solid surface. This mechanism described the interactions between a liquid and a solid surface and was quantified, at mechanical equilibrium, by means of the apparent contact angle $\theta$, also referred to as static contact angle [5]. Liquid-repellent surfaces exhibited increased resistance to wetting which translated into large values for $\theta$. Controlling wettability of a surface proved to be a valuable tool for the design of numerous engineering applications including hydrophobic [6, 7] or oleophobic [8] non-wetting textiles, anti-fogging [9] and anti-icing [10] technologies, optimized buoyancy [11] and associated flow improvement [12], as well as antibiofouling [13] and water collection from fog [14].

Despite numerous applications invoking the theoretical underpinnings of wettability, understanding and controlling it remains largely still elusive thereby constituting a perpetual struggle for scientist, in which, luckily, they are not alone. To the observant enough and with keen perceptiveness, nature proved to be a valuable ally exemplifying numerous cases of 
biological surfaces with remarkable wetting behavior. In the relatively recently spawned and rapidly developing fields of biomimetics and bionics, applications were engineered with functions emulating everyday behavior of such biological systems. Prominent examples of such influential bioinspired-surfaces and resulting technological applications included nonreflective surfaces utilizing the "moth-eye” effect [15], inspection robots with highly adhesive surfaces making use of the "gecko-effect" [16, 17], self-cleaning window panes using the "lotus leaf effect” [18], solid bodies exhibiting improved flowability owed to reduced drag by exploiting the "shark skin effect" [19, 20], as well as numerous other emerging applications related to energy conversion and conservation [21].

The difficulty to comprehend wettability fully was, in large part, attributed to the great number of parameters contributing to the intricacy of the phenomenon, including surface chemistry [22, 23], surface roughness [24, 25], temperature [26], mechanical loading [27], crystallinity [28] and applied electric field [29, 30]. Chemistry modifications of the surface, albeit promising, proved to have rather limited effect on wettability [31]. In contrast, changes in the surface topography appeared a more efficient way for manipulating wettability, even for the design of oil-repellent surfaces [32]. For even more pronounced control over wettability, very recently, topography modifications were combined efficiently with chemical treatment of various surfaces including cotton [33, 34], steel [35] and synthetic leather [36]. Further examples include trichloro $(1 \mathrm{H}, 1 \mathrm{H}, 2 \mathrm{H}, 2 \mathrm{H}$-perfluorooctyl) silane (PFOCTS)-modified cobalt structures for creating omniphobic surfaces [37], Cd-Si co-doped $\mathrm{TiO}_{2}$ thin films examining the synergistic effect of co-dopants and calcination temperature on wettability [38], as well as polymer nanocomposite surfaces comprising $\mathrm{Au} / \mathrm{ZnO}$ nanoparticles randomly dispersed in a cellulose acetate polymer matrix [39]. A detailed compilation of substrate coatings and associated fabrication techniques can be found in the comprehensive work of B. Bhushan and S. Martin [40].

In the general context of tinkering surface topography, surface roughness was of paramount importance [32, 41]. Extensive studies on biological surfaces, such as the leaves of Ginkgo biloba [25] and Nelumbo nucifera (lotus) [42] plants elaborated on the effect of roughness on overall nonwetting capabilities. The superhydrophobic traits of these biological surfaces were attributed initially to their distinct surface roughness which theoretically rendered them in a metastable state. This assumption was later on confirmed in the pioneering works of Marmur [43] and Nosonovsky [44]. Subsequently, an analysis entailing Gibbs surface free energy minimization in various solid-liquid-air systems showed that wetting states were distinct and 
could indeed be metastable and rather long-lived [45]. Besides surface roughness, biological surfaces often exhibited hierarchical organization, i.e., multiple levels of surface topographies placed on top of each other, which, in turn, was responsible for increased surface roughness owed to larger surface-to-volume ratio. A characteristic example was the morphology of the lotus leaves comprising nanoscale roughness superimposed over microscale bumps [46]. Hierarchical topographies in biological surfaces were, in many instances, identified to promote liquid repellency [47, 48].

Engineered surfaces, on the other hand, differed, in many cases considerably, from biological surfaces due to multilevel hierarchical organization customarily present in the latter. This was because implementing multilevel topographies of multiplicity larger than two on engineered surfaces constituted a formidable technological challenge. Interestingly, non-hierarchical surfaces, which, however, retained some degree of roughness, displayed superhydrophobicity [49]. In addition, water-repelling surfaces were produced without necessarily featuring hierarchical topography $[50,51]$. Therefore, in total, roughness was seemingly more important than hierarchical organization (multilevel topography), the latter being, however, beneficial for enhanced liquid-repellency.

Modelling wettability played a decisive role in the design of surface topographies with tailored properties, as well as in the interpretation of experimental observations. Ever since the inaugural modelling attempts undertaken by Thomas Young in 1805 [52], followed by Wenzel [53] and eventually by Cassie and Baxter [54], numerous theoretical works emerged [55-60] thereby manifesting the great technological potential associated with increased wettability control. Without any loss of generality and for simplicity, most of these models considered only two-dimensional (2D) systems, implying that the surface texture extended to infinity along the remaining dimension forming thus a semi-infinite three-dimensional (3D) representation. This practice was customary and was shown to produce results in gratifying agreement with realistic 3D measurements [61, 62]. Most of these works made use of the classical CassieBaxter (CB) model or some variation of it, assuming thus that the liquid-air interface was a straight line. Usually, this held true because of the typically small distance between neighboring surface irregularities.

Complementing seminal works trying to address this issue [63-66], in our recent study [67], we revoked this constraint by deriving the formalism of a refined CB model which, nonetheless, considered curved liquid-air interfaces on various single- and two-level 
topographies. Direct comparison between our model and the original CB model revealed that the latter overpredicted substantially the contact angle, while it failed to capture the anticipated trends between contact angle and roughness. Because of this deviating behavior, the original CB model did not capture the transitions from the CB state to the Wenzel and Young states with decreasing surface roughness.

\section{Materials and Methods}

Throughout this work, the basis of the calculations was our refined CB model derived and applied for the predictions of surface topographies exhibiting superomniphobic traits [67]. The heart of our methodology lay in the consideration of realistic curved liquid-air interfaces separating trapped air from supernatant liquid. The radius of the curved meniscus was defined with the help of the capillary length $l_{\text {cap }}=\sqrt{\gamma_{\text {la }} / \rho g}$, which was, in turn, a function of the liquid surface tension $\gamma_{\text {la, }}$, the liquid density $\rho$ and the gravitational acceleration $g$. The curvature of the liquid-air interface was then quantified by considering the sagging (or protrusion) height $h$ $=l / l_{\text {cap }}$, where $l$ was a characteristic topography-dependent length. For all considered singlelevel surface topographies, $l$ was defined accordingly [67].

By observing the original CB model, $\theta^{\mathrm{CB}}=f_{\mathrm{sl}} \cos \theta^{\mathrm{Y}}-f_{\text {la, }}$, where $f_{\mathrm{sl}}$ and $f_{\text {la }}$ describe the area fractions of the solid-liquid and liquid-air interfaces, respectively, and the superscript $\mathrm{Y}$ denotes the contact angle by virtue of Young's relation [52], it becomes obvious that the calculation of $\theta^{\mathrm{CB}}$ depends on $f_{\mathrm{sl}}$ and $f_{\mathrm{la}}$, which, in turn, depend on the surface topography type and the shape of the liquid-air interface. Correspondingly, in our previous work [67], analytical expressions for $f_{\mathrm{sl}}$ and $f_{\text {la }}$ were derived for the 2D pillars, fiber and sinusoidal surface topographies illustrated in figures $1 \mathrm{a}, 1 \mathrm{~b}$ and 1c, respectively. In addition, a generic algebraic expression for modeling multilevel hierarchical roughness of arbitrarily large multiplicity was derived based on information on single-level roughness. This expression was employed here to predict the water contact angle on hierarchical two- and three-level topographies.

Our model requires as input physicochemical parameters of the wetting liquid (density $\rho$ and surface tension $\gamma_{\mathrm{la}}$ ), Young contact angle $\theta^{\mathrm{Y}}$ of the wetting liquid on a completely flat and smooth surface made of the same material as the rough surface, as well as type and dimensionality of surface topography. The Young contact angle can be measured experimentally, or, alternatively, it can be computed by virtue of the Young equation $\cos \theta^{\mathrm{Y}}=$ 
$\left(\gamma_{\mathrm{s}}-\gamma_{\mathrm{sl}}\right) / \gamma_{\mathrm{la}}$, where $\gamma_{\mathrm{s}}$ and $\gamma_{\mathrm{la}}$ are the solid surface energy and liquid surface tension terms, respectively, which can be measured experimentally or be found in material databases. The remaining interfacial surface energy term $\gamma_{\text {sl }}$ cannot be measured directly and thus needs to be computed by using any of the conventional Fowkes [68], Zisman [69] or Owens-Wendt [70] theoretical approximations, with the latter being slightly preferential over the others. Should this be the case, information on the polar and dispersive components of $\gamma_{\text {la }}$ and $\gamma_{\mathrm{s}}$ is required. Here, the Young contact angle was measured experimentally or, where applicable, was found in the literature (see below) and thus there was no need to resort to measuring the polar and dispersive parts of $\gamma_{\text {la }}$ and $\gamma_{\mathrm{s}}$.

The objective of the current work was twofold: on one side, we extended the formalism of our refined CB model from semi-infinite 2D to 3D topographies, whereas, on the other side, we tested and validated our model, in its entirety, by comparing directly model predictions with experimental data for various combinations of surface topographies, hierarchy levels and surface materials. Accordingly, area fractions $f_{\text {sl }}$ and $f_{\text {la }}$ were derived for a new 3D surface topography featuring 3D pillars with square cross section. A schematic illustration of this new single-level topography, along with its characteristic geometric details are shown in figure 1(d). The resulting area fraction relations for this topography are as follows (see supplementary material for derivation):

$$
\begin{aligned}
& f_{\mathrm{sl}}=\frac{W^{2}}{D_{\mathrm{p}}^{2}} \\
& f_{\mathrm{la}}=\frac{2 l_{\mathrm{arc}} W+\left(l_{\mathrm{arc}}\right)^{2}}{D_{\mathrm{p}}^{2}}
\end{aligned}
$$

where $W$ and $D_{\mathrm{p}}$ represent the width and unit cell distance (see figure $1(\mathrm{~d})$ ) and $l_{\text {arc }}$ is the numerator of the corresponding fla relation for $2 \mathrm{D}$ pillars as it was derived in our recent work for different wetting cases, i.e., wetting liquid touches (wets) the surface base level or not [67]. To validate our model, totally eleven single-level surface topographies of various dimensions and types were considered in full compliance with the three basic surface topographies introduced originally [67], namely 2D flat-top pillars (figure 1(a)), fibers with circular crosssection (figure 1(b)) and sinusoids (figure 1(c)). To further validate the ability of our model to capture accurately wetting of surfaces with 3D texture, additionally eight 3D-pillar topographies of various dimensions were used for a total of nineteen single-level surface topographies (2D and 3D). Figures 1(e)-(h) describe representative experimental surfaces 
displaying 2D-pillar, sinusoidal and 3D-pillar topography, respectively. Furthermore, to validate our model predictions related to multilevel topography, twelve two-level and one three-level hierarchical surfaces were studied. Representative 2D schematic illustrations of the two- and three-level surface topographies considered here are shown in Figure 2. Accordingly, figure 2a illustrates the "pillars on sinusoids" topography corresponding to structures 20-22 in Table 2, figure 2b shows the "sinusoids on sinusoids" topography describing structures 23-31 in Table 2, whereas figure 2c displays the "triple sinusoidal” topography of structure 32 in Table 2. A detailed description of the characteristic geometrical parameters of the experimental single- and multilevel surface topographies considered here is provided in Tables 1 and 2, respectively. Given the great technological importance of surfaces exhibiting patterns of diverse length scales, caution was taken here that a broad spectrum of characteristic lengths was spanned ranging from a few hundreds of micrometers to a few tens of nanometers (cf. Tables 1 and 2). This corroborates the generic validity and applicability of our model.

Throughout this work, the wetting liquid was water having density $\rho$ and surface tension $\gamma_{\text {la }}$ at ambient conditions equal to $997 \mathrm{~kg} / \mathrm{m}^{3}$ and $72.8 \mathrm{mN} / \mathrm{m}$, respectively. To extract conclusions on the generic character of our model, a rich variety of surface materials was considered involving silanized silicon wafers, poly(lactic-co-glycolic) acid (PLGA) comprising 85\% polylactic acid (PLA) and 15\% polyglycolic acid (PGA), cyclic olefin copolymer (COC), polydimethylsiloxane (PDMS), aluminium and an epoxy-based shape memory polymer (SMP). Structures 1-3 in Table 1 were fabricated on silicon wafers using standard UVlithographic techniques. Briefly, silicon wafers were spin-coated with a positive photoresist and exposed to UV light in an EVG 620 mask aligner using a designed chromium-on-glass photomask. After photoresist development, a Deep Reactive Ion Etching (DRIE) process was carried out in an Oxforf Plasmalab 80+ machine to etch the silicon in the areas not covered by the photoresist. After etching, the remaining photoresist was removed by successive sonication in acetone, IPA and deionized water.

Then, the wafer was fluorinated by exposure for $30 \mathrm{~min}$ to a saturated atmosphere of vapor PFOCTS. Structures 9-11 were fabricated using a picosecond laser Nd:YVO4 (3D MicroMac Microstruct) with pulses of 10 ps and wavelength of 1064, 532 and 355 nm, respectively. Therein, the beam diameter was smaller than $30 \mu \mathrm{m}$. Contact angle measurements for structures 1-3 and 9-11 were performed on a Surftens Universal (OEG) goniometer. Analogously, polymer-based surface topographies 4 and 12-14 were fabricated by hot embossing on a Jenoptik HEX 03 using a silicon wafer with test patterns manufactured by photolithography and subsequent etching. Hot embossing was carried out at $100{ }^{\circ} \mathrm{C}$ for PLGA and at $120^{\circ} \mathrm{C}$ for 
COC. Water contact angle measurements were performed on a Krüss drop shape analyzer (DSA 100).. For all surfaces constructed in the context of this work (cf. Table 1), the Young contact angle of water was measured with the help of the goniometers reported above. 
(a)

(b)
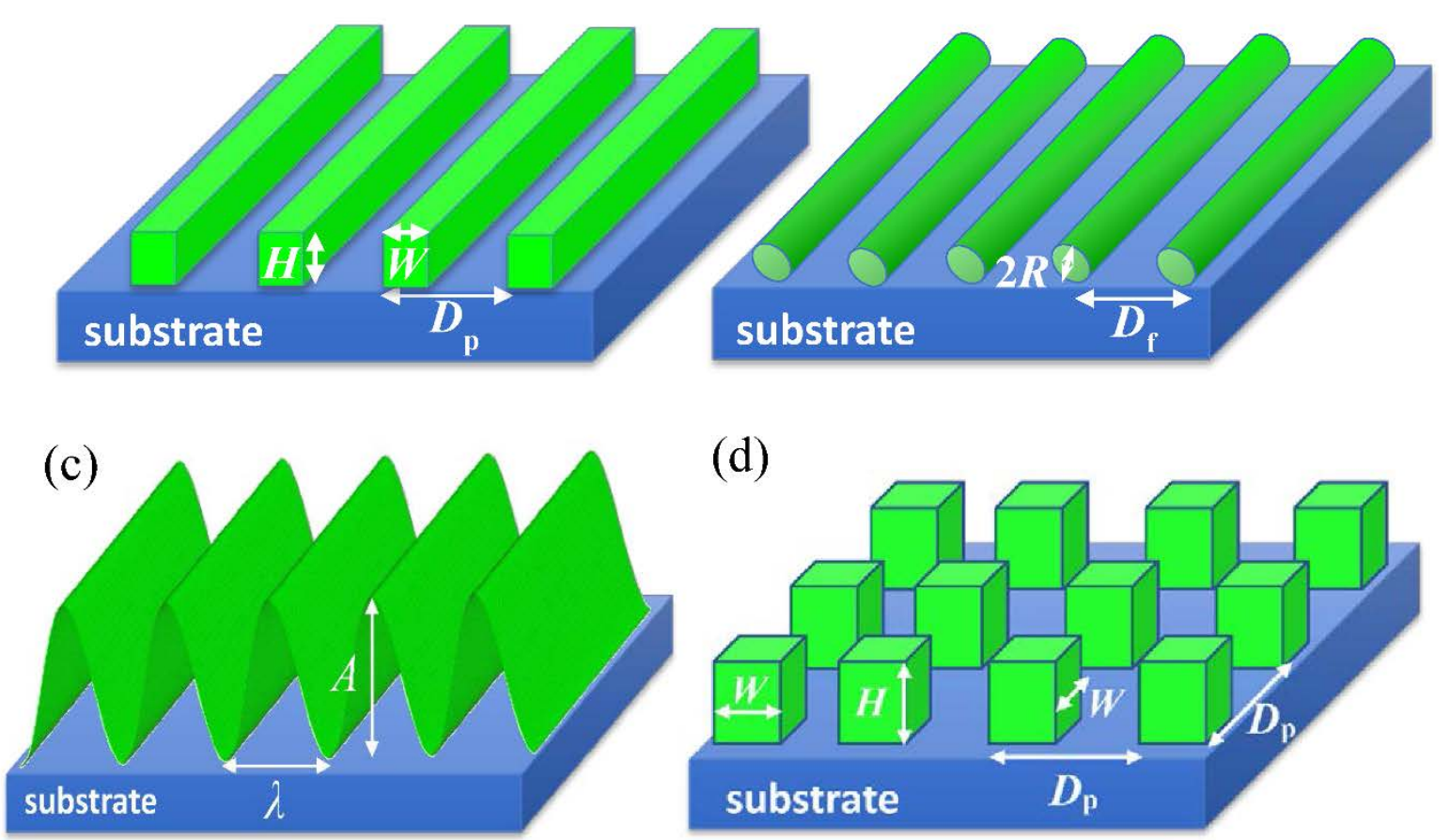

(d)

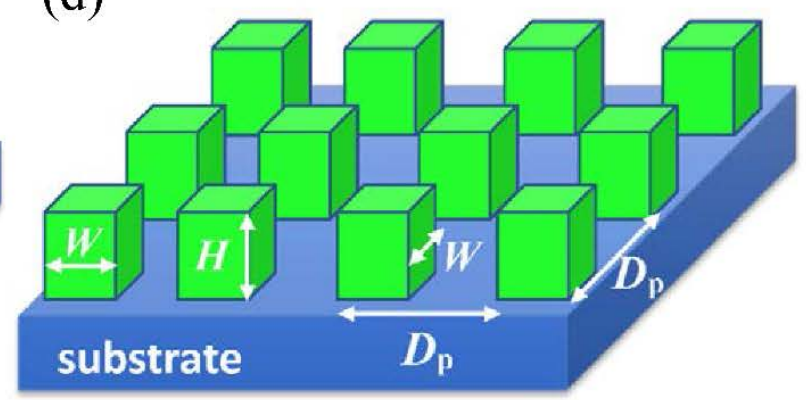

(e)

(f)
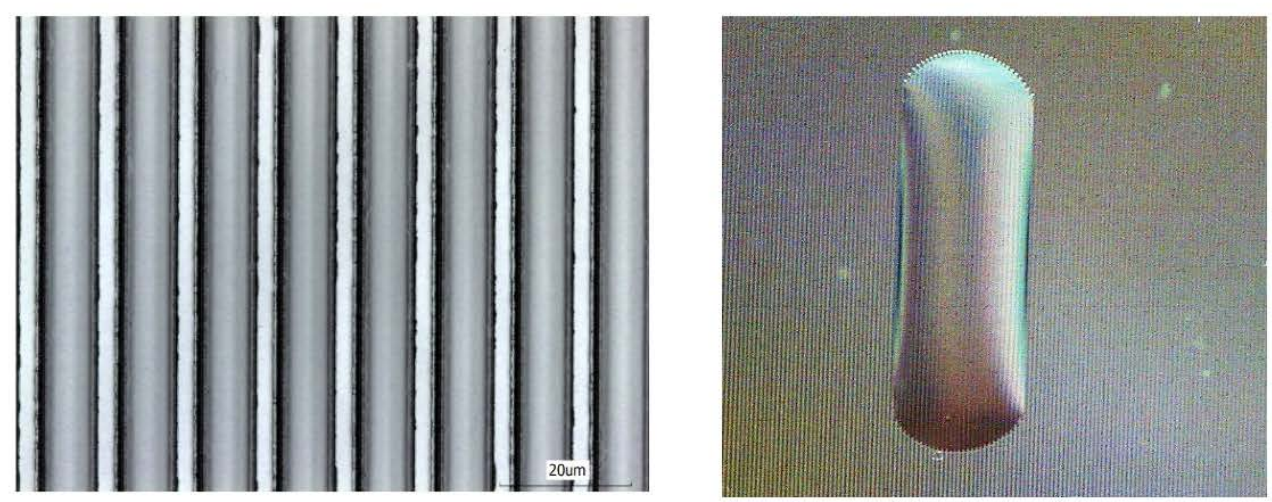

(g)

(h)
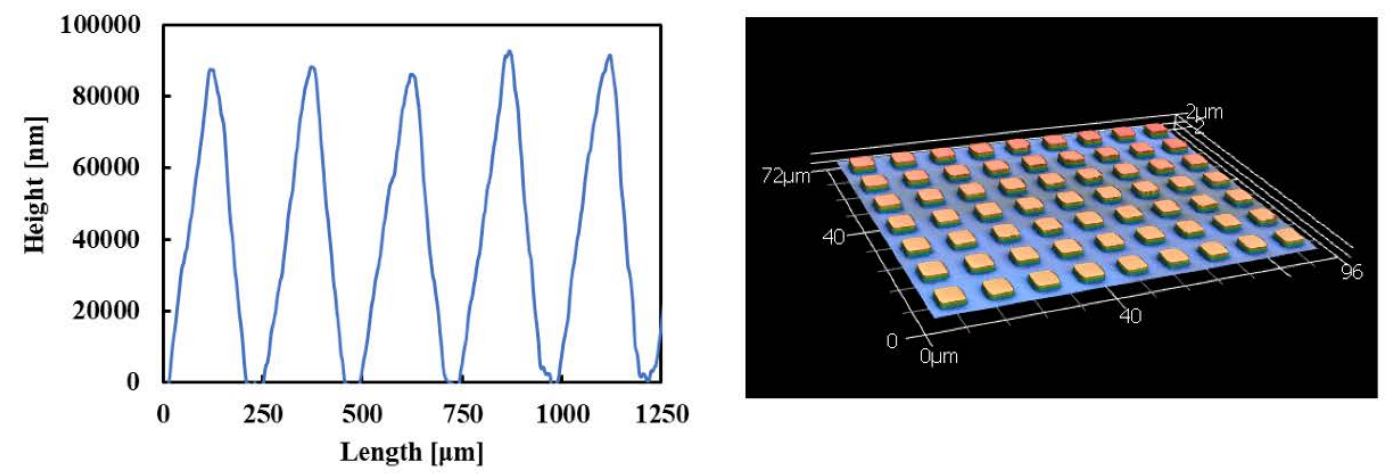

Figure 1. Schematic illustration of single-level (a) 2D-pillar, (b) fiber, (c) sinusoidal and (d) 3D-pillar topographies. Relevant geometric parameters are also shown. (e) Confocal laser scanning microscopy (CLSM) top view of a representative 2D-pillar topography corresponding 
to structure 4 in table 1, (f) pronounced anisotropic wetting by water (picture taken from underneath the wetted sample of part (e), (g) Profile of an indicative sinusoidal topography corresponding to structure 9 in table 1 measured by a mechanical profilometer (Veeco Dektrak8 with $0.7 \mu \mathrm{m}$ tip) and (h) CSLM 3D view of a representative 3D-pillar topography corresponding to structure 13 in table 1.

(a)

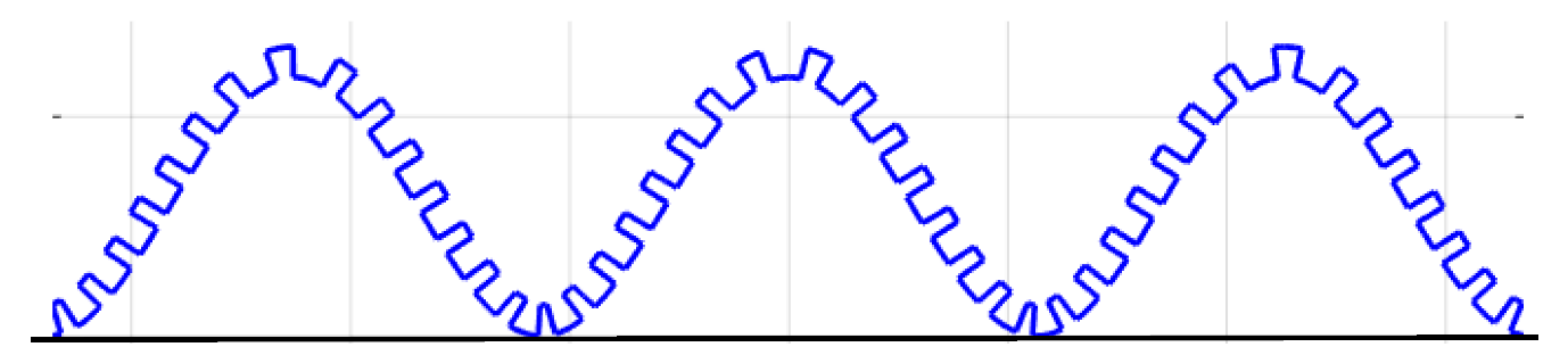

(b)
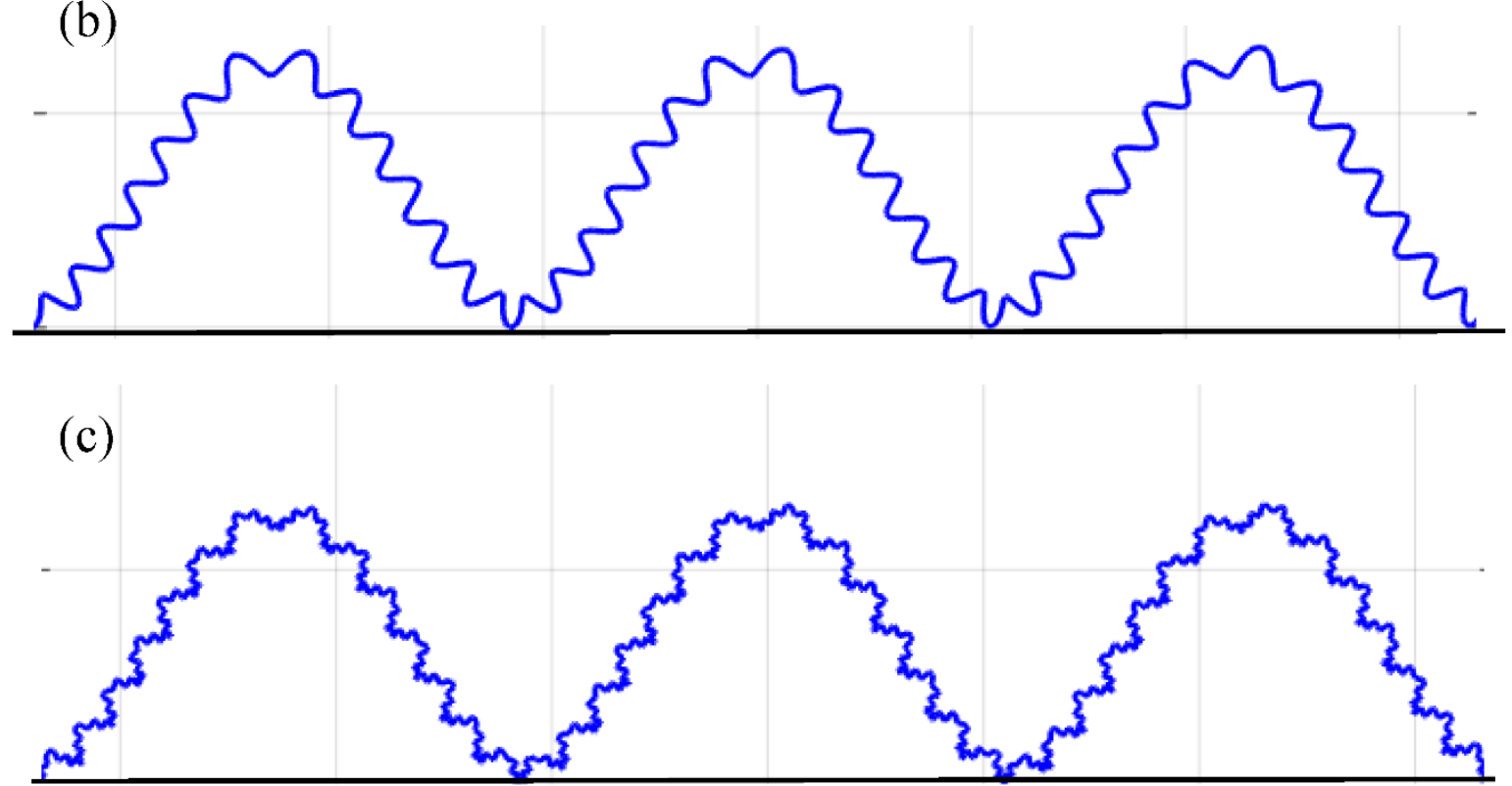

Figure 2. Representative 2D schematic illustrations of the hierarchical two-level a) pillars on sinusoids, b) sinusoids on sinusoids and c) three-level triple sinusoidal surface topographies described in Table 2.

Table 1. Description of single-level surface topographies considered here. Symbols $H, W$ and $D_{\mathrm{p}}$ correspond to pillar height, width and unit cell distance, respectively, whereas $R$ and $D_{\mathrm{f}}$ describe the fiber radius and unit cell distance, respectively. Finally, $A$ and $\lambda$ represent the 
amplitude and wavelength of the sinusoidal topography, respectively. Experimental Young's contact angle $\theta^{\mathrm{Y}}$ of water on each surface is also reported.

\begin{tabular}{|c|c|c|c|c|c|}
\hline $\begin{array}{c}\text { Structure } \\
\#\end{array}$ & Topography & Dimensions $[\mu \mathrm{m}]$ & $\begin{array}{l}\text { Surface } \\
\text { material }\end{array}$ & $\theta^{\mathrm{Y}}\left[{ }^{0}\right]$ & Ref. \\
\hline 1 & 2D Pillars & $H=0.253, W=25, D_{\mathrm{p}}=50$ & $\begin{array}{c}\text { silanized } \\
\text { silicon } \\
\text { wafer }\end{array}$ & 116.7 & this work \\
\hline 2 & 2D Pillars & $H=0.253, W=50, D_{\mathrm{p}}=100$ & $\begin{array}{c}\text { silanized } \\
\text { silicon } \\
\text { wafer }\end{array}$ & 116.7 & this work \\
\hline 3 & 2D Pillars & $H=0.253, W=100, D_{\mathrm{p}}=200$ & $\begin{array}{c}\text { silanized } \\
\text { silicon } \\
\text { wafer }\end{array}$ & 116.7 & this work \\
\hline 4 & 2D Pillars & $H=4.4, W=3.4, D_{\mathrm{p}}=12$ & PLGA & 72.7 & $\begin{array}{c}\text { this work } \\
\&[71]\end{array}$ \\
\hline 5 & 2D Pillars & $H=10, W=45.5, D_{\mathrm{p}}=90.6$ & $\mathrm{COC}$ & 88.3 & {$[72]$} \\
\hline 6 & fibers & $R=18, D_{\mathrm{f}}=79$ & PDMS & 105 & {$[8]$} \\
\hline 7 & fibers & $R=12.5, D_{\mathrm{f}}=63$ & PDMS & 105 & {$[8]$} \\
\hline 8 & fibers & $R=14, D_{\mathrm{f}}=78$ & PDMS & 105 & {$[8]$} \\
\hline 9 & sinusoidal & $A=86, \lambda=225$ & aluminium & 67.1 & this work \\
\hline 10 & sinusoidal & $A=96, \lambda=280$ & aluminium & 67.1 & this work \\
\hline 11 & sinusoidal & $A=90, \lambda=480$ & aluminium & 67.1 & this work \\
\hline 12 & 3D Pillars & $H=1.6, W=2.6, D_{\mathrm{p}}=3.9$ & $\mathrm{COC}$ & 93 & this work \\
\hline 13 & 3D Pillars & $H=1.7, W=6.0, D_{\mathrm{p}}=10.4$ & $\mathrm{COC}$ & 93 & this work \\
\hline 14 & 3D Pillars & $H=1.8, W=3.9, D_{\mathrm{p}}=6.6$ & $\mathrm{COC}$ & 93 & this work \\
\hline 15 & 3D Pillars & $H=8.6, W=44.7, D_{\mathrm{p}}=90.6$ & $\mathrm{COC}$ & 88.3 & {$[72]$} \\
\hline 16 & 3D Pillars & $H=8.0, W=45.5, D_{\mathrm{p}}=90.6$ & $\mathrm{COC}$ & 88.3 & {$[72]$} \\
\hline 17 & 3D Pillars & $H=10.0, W=10.0, D_{\mathrm{p}}=20.0$ & SMP & 89 & {$[73]$} \\
\hline
\end{tabular}




\begin{tabular}{|c|c|c|c|c|c|}
\hline 18 & 3D Pillars & $H=10.0, W=10.0, D_{\mathrm{p}}=30.0$ & SMP & 89 & [73] \\
\hline 19 & 3D Pillars & $H=10.0, W=10.0, D_{\mathrm{p}}=40.0$ & SMP & 89 & [73] \\
\hline
\end{tabular}

Table 2. Description of hierarchical multilevel surface topographies considered here. Symbols are identical to Table 1. Subscripts 1, 2 and 3 (where applicable) denote different levels starting from the finest towards coarser ones. Experimental Young's contact angle $\theta^{\mathrm{Y}}$ of water on each surface is also reported.

\begin{tabular}{|c|c|c|c|c|c|}
\hline $\begin{array}{c}\text { Structure } \\
\#\end{array}$ & Topography & Dimensions $[\mu \mathrm{m}]$ & $\begin{array}{l}\text { Surface } \\
\text { material }\end{array}$ & $\theta^{\mathrm{Y}}\left[{ }^{\mathrm{o}}\right]$ & Ref. \\
\hline 20 & $\begin{array}{l}\text { pillars on } \\
\text { sinusoids }\end{array}$ & $\begin{array}{c}H=0.15, W=0.3, D_{\mathrm{p}}=0.5 \\
A=4.4, \lambda=31\end{array}$ & PDMS & 110 & [74] \\
\hline 21 & $\begin{array}{l}\text { pillars on } \\
\text { sinusoids }\end{array}$ & $\begin{array}{c}H=0.35, W=0.2, D_{\mathrm{p}}=0.5 \\
A=4.4, \lambda=31\end{array}$ & PDMS & 110 & [74] \\
\hline 22 & $\begin{array}{l}\text { pillars on } \\
\text { sinusoids }\end{array}$ & $\begin{array}{c}H=0.7, W=0.15, D_{\mathrm{p}}=0.5 \\
A=4.4, \lambda=31\end{array}$ & PDMS & 110 & [74] \\
\hline 23 & $\begin{array}{l}\text { sinusoids on } \\
\text { sinusoids }\end{array}$ & $\begin{array}{c}A_{1}=0.275, \lambda_{1}=0.822 \\
A_{2}=0.6, \lambda_{2}=25\end{array}$ & PDMS & 119 & [75] \\
\hline 24 & $\begin{array}{l}\text { sinusoids on } \\
\text { sinusoids }\end{array}$ & $\begin{array}{c}A_{1}=0.322, \lambda_{1}=0.774 \\
A_{2}=2.75, \lambda_{2}=39\end{array}$ & PDMS & 119 & [75] \\
\hline 25 & $\begin{array}{l}\text { sinusoids on } \\
\text { sinusoids }\end{array}$ & $\begin{array}{c}A_{1}=0.305, \lambda_{1}=0.79 \\
A_{2}=3.5, \lambda_{2}=39\end{array}$ & PDMS & 119 & [75] \\
\hline 26 & $\begin{array}{l}\text { sinusoids on } \\
\text { sinusoids }\end{array}$ & $\begin{array}{c}A_{1}=0.311, \lambda_{1}=0.825 \\
A_{2}=4.35, \lambda_{2}=34\end{array}$ & PDMS & 119 & [75] \\
\hline 27 & $\begin{array}{l}\text { sinusoids on } \\
\text { sinusoids }\end{array}$ & $\begin{array}{c}A_{1}=0.247, \lambda_{1}=0.77 \\
A_{2}=2.5, \lambda_{2}=16.7\end{array}$ & PDMS & 119 & [75] \\
\hline 28 & $\begin{array}{l}\text { sinusoids on } \\
\text { sinusoids }\end{array}$ & $\begin{array}{c}A_{1}=0.297, \lambda_{1}=0.8 \\
A_{2}=4.5, \lambda_{2}=25.8\end{array}$ & PDMS & 119 & [75] \\
\hline 29 & $\begin{array}{l}\text { sinusoids on } \\
\text { sinusoids }\end{array}$ & $\begin{array}{c}A_{1}=0.27, \lambda_{1}=0.776 \\
A_{2}=3.75, \lambda_{2}=20\end{array}$ & PDMS & 119 & [75] \\
\hline 30 & $\begin{array}{l}\text { sinusoids on } \\
\text { sinusoids }\end{array}$ & $\begin{array}{c}A_{1}=0.315, \lambda_{1}=0.815 \\
A_{2}=4.5, \lambda_{2}=23.3\end{array}$ & PDMS & 119 & [75] \\
\hline 31 & $\begin{array}{l}\text { sinusoids on } \\
\text { sinusoids }\end{array}$ & $\begin{array}{c}A_{1}=0.267, \lambda_{1}=0.75 \\
A_{2}=4.2, \lambda_{2}=21.4\end{array}$ & PDMS & 119 & [75] \\
\hline 32 & $\begin{array}{c}\text { triple } \\
\text { sinusoidal }\end{array}$ & $\begin{array}{c}A_{1}=0.075, \lambda_{1}=0.15 \\
A_{2}=0.45, \lambda_{2}=0.9 \\
A_{3}=5, \lambda_{3}=10\end{array}$ & PDMS & 110 & {$[76]$} \\
\hline
\end{tabular}




\section{Results and Discussion}

\subsection{Single-level topographies}

Figures 3 and 4 illustrate comparisons between calculated contact angles and their experimental counterparts for the nineteen single-level surface topographies listed in Table 1. In particular, figure 3 shows contact angle comparisons for 2D pillars, fibers, and sinusoidal topographies, whereas figure 4 for 3D pillars. For the 2D pillars case, depicted in figure 3, the agreement between experiment and model was gratifying resulting to an average error of about $6 \%$. This was, at least partially, attributed to the inherent assumption of our model pertaining to pinning of the liquid at the corners of the pillars. This assumption hampered the droplet from intruding in the free space between neighboring pillars thus causing artificially increased contact angles as evinced by figure 3. Nonetheless, this discrepancy lay for three of the totally five tested structures displaying this topography within the uncertainties of the experimental data. The model results for the fiber topography, shown in figure 3, were substantially closer to their experimental counterparts giving rise to an average error below 3\%. Clearly, figure 3 shows that the attained contact angles for the fiber topography were larger than the ones for 2D pillars, thereby corroborating previous modeling observations and associated trends [67] relating this behavior with the re-entrant geometry of the fiber topography.

The calculated contact angles for the sinusoidal topography were substantially smaller than the previous two single-level topographies, namely 2D pillars and fibers. This observation becomes apparent by juxtaposing the three topographies shown in figure 3 and is in excellent agreement with recent theoretical predictions identifying fibers as the most efficacious amongst the three single-level topographies for achieving high contact angles, followed, in order of efficiency, by 2D pillars and finally sinusoidal surfaces [67]. Figure 3 shows that the agreement between experimental measurements and model predictions for sinusoidal topographies was remarkably good exhibiting an average error of only $4.6 \%$.

Next, consideration of actual 3D surface topographies, instead of simplified semi-infinite 2D structures, resulted in increased water contact angles and thus enhanced water repellency. This behavior is illustrated in figure 4 for the 3D-pillars case and was attributed to an augmented surface-to-volume ratio, generally more pronounced in 3D structures than in commensurate 2D topographies, inducing thereby increased surface roughness and thus enhanced liquidrepellency. The predicted contact angles were, once again, in reasonable accord with experimental observations displaying average deviations of not more than $4.5 \%$. Taken all together, predicted contact angles for the four types of single-level topographies considered 
here (see Table 1) deviated, on average, from experimental measurements by less than $5 \%$. This discrepancy was, for most surface topographies examined here, within the statistical uncertainties of experimental data.

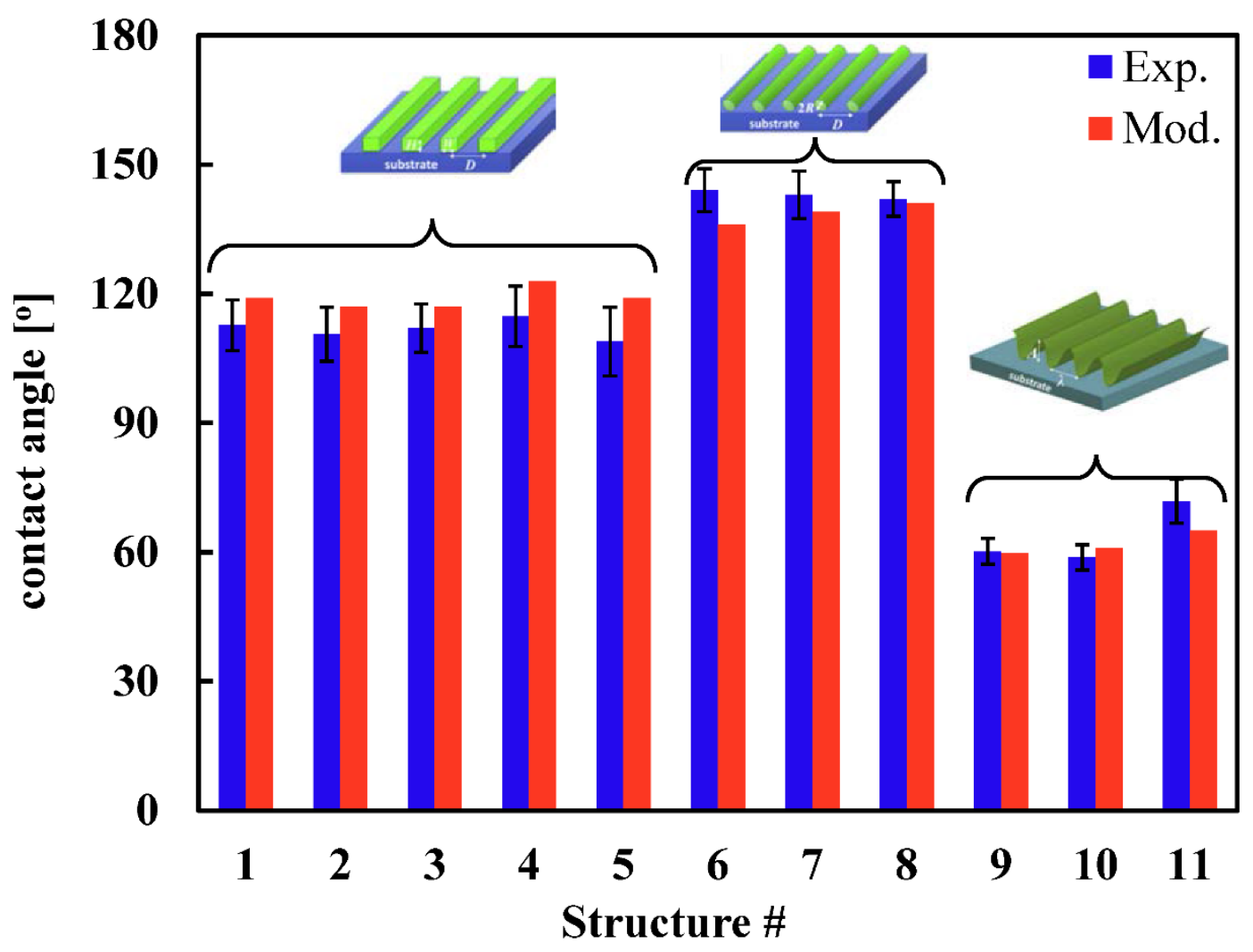

Figure 3. Contact angle comparison between model results (red) and experimental data (blue) for eleven single-level topographies: structures 1-5, 6-8 and 9-11 correspond to 2D pillars, fibers and sinusoid topographies, respectively (see Table 1 and Ref. [67]). 


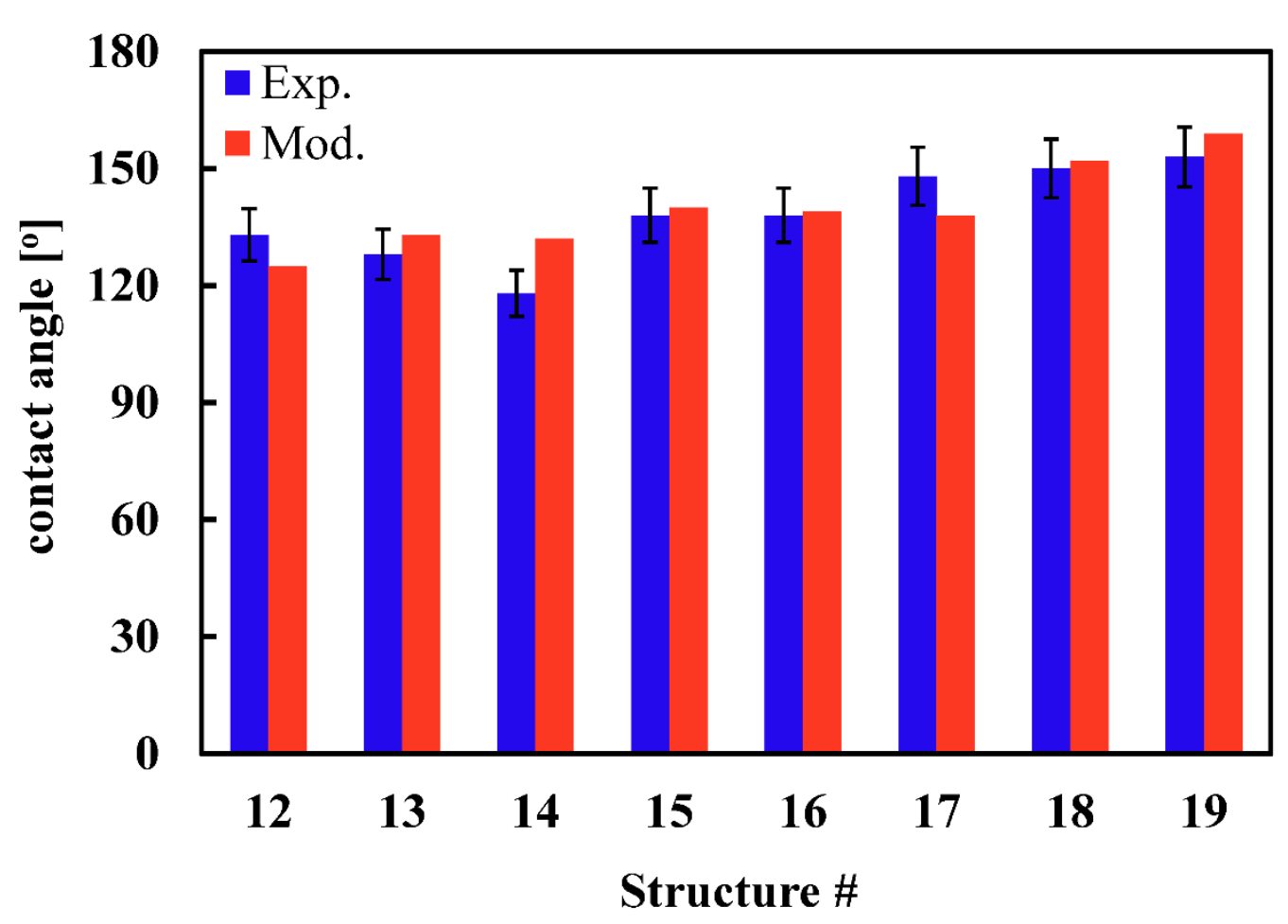

Figure 4. Contact angle comparison between model results (red) and experimental data (blue) for eight single-level topographies with 3D-pillars texture (see Table 1 and figure 1).

\subsection{Multilevel topographies}

It is well established in the literature that multilevel hierarchical surface topographies induced enhanced liquid repellency. Figure 5 validates this consensus by illustrating calculated and experimentally measured contact angles for two types of two-level topography. For most structures in figure 5, contact angles lay in the vicinity of $140^{\circ}$, with two surfaces exhibiting superhydrophobic behavior $\left(\theta>150^{\circ}\right)$. Structures 20-22 in figure 5 correspond to two-level topographies featuring pillars (fine level) on top of sinusoids (coarse level), wherein the two levels differed in size by at least one order of magnitude (see table 2). This observation validates previous theoretical findings arguing that a size difference of at least one order of magnitude between the superimposed surface patterns was required for achieving superhydrophobicity [67]. Notwithstanding the significance and influence of multilevel hierarchical structures on liquid repellency, the construction of such surfaces poses substantial technological challenges. This is due to the intrinsic technological difficulties associated with manufacturing of surface details on the submicron or even nanoscale.

Structures 23-31 in figure 5 correspond to "sinusoids on sinusoids" two-level topographies giving rise to contact angles consistently smaller than the ones for "pillars on sinusoids" 
surfaces. Given that the surface material was in both cases the same (PDMS), the coarse level was of the same type (sinusoids) and, more or less, on the same order of magnitude (table 2), we discerned that the behavior of two-level topographies was greatly influenced by the upper (finer) level. According to the single-level results reported in Section 3.1, 2D-pillar surfaces exhibited, in general, larger contact angles than sinusoidal topographies. The behavior shown in figure 5 is, therefore, consistent with this observation. For both types of two-level topography considered here, the calculated contact angles were in notable accord with their experimental counterparts. The average deviation for the "pillars on sinusoids" and "sinusoids on sinusoids” topographies amounted to $4.7 \%$ and $3.2 \%$, respectively, resulting in an average deviation of $4 \%$ for the two-level topographies.

Finally, figure 6 shows the comparison between the predicted and the experimental contact angle for a three-level topography with three levels of sinusoid, each having dimensions that differed by one order of magnitude from the next one (table 2). Superhydrophobicity was observed here as well, further validating the general belief that multilevel topographies enable increased liquid-repellency. Indeed, this becomes clear by comparing the contact angles for sinusoidal single-, two- and three-level topographies shown in figures 3 (structures 9-11), 4 (structures 23-31) and 5, respectively. The addition of a second level of sinusoidal patterns almost doubled the observed contact angle, while incorporation of a third sinusoidal level brought about an additional $12.5 \%$ contact angle increase. Again, our model predicted for this three-level surface topography a contact angle that deviated only by $3.1 \%$ from the experimentally measured value, well within the statistical uncertainty of the experimental measurement. Taken all together, figures 5, 6 and the discussion therein confirmed the ability of our model to predict very accurately the wetting behavior of surfaces exhibiting multilevel hierarchical topographies. 


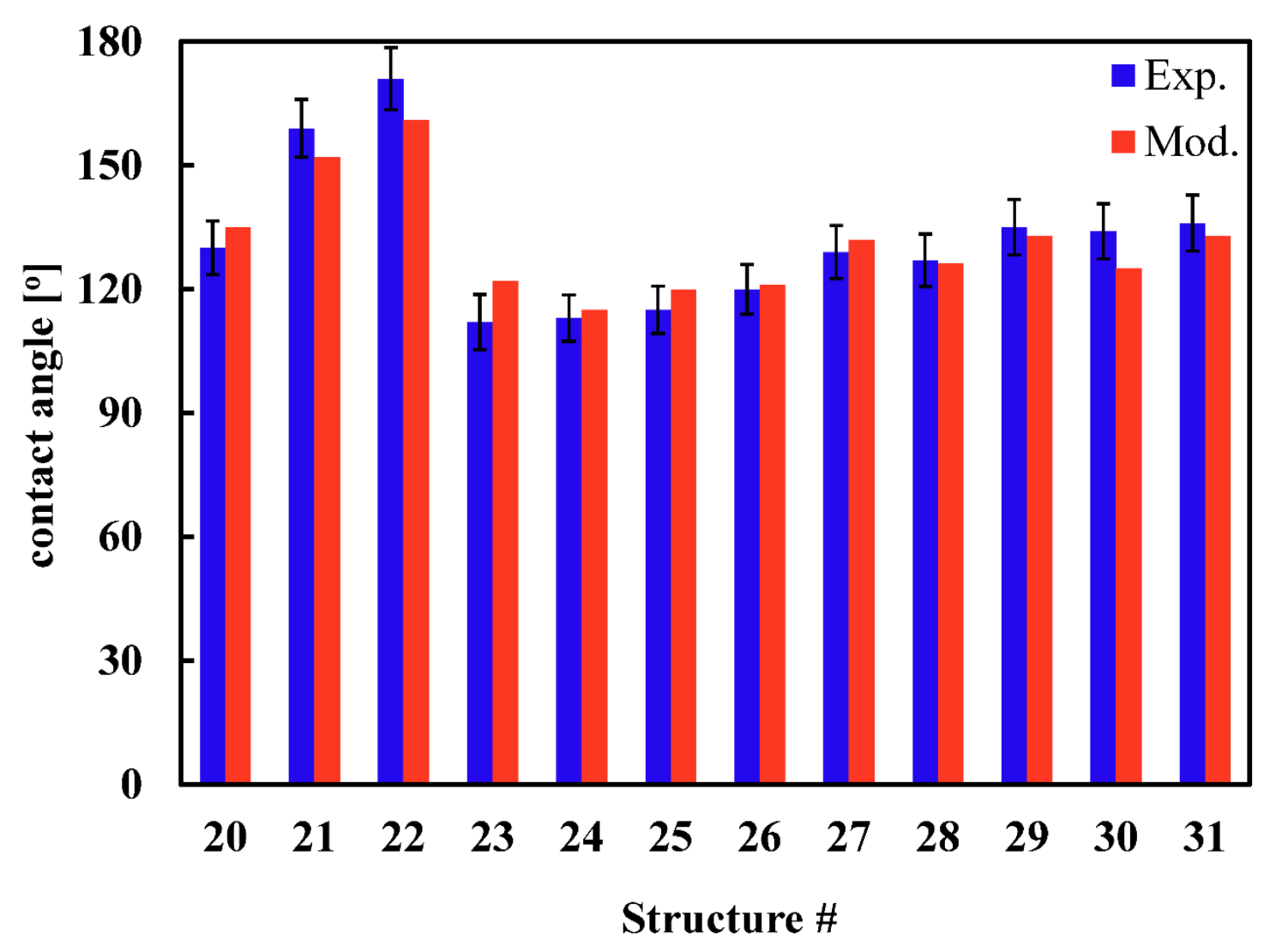

Figure 5. Contact angle comparison between model results (red) and experimental data (blue) for twelve two-level surface topographies: Structures 20-22 describe "pillars on sinusoids", whereas structures 23-31 correspond to "sinusoids on sinusoids" topographies. (see Table 2). 


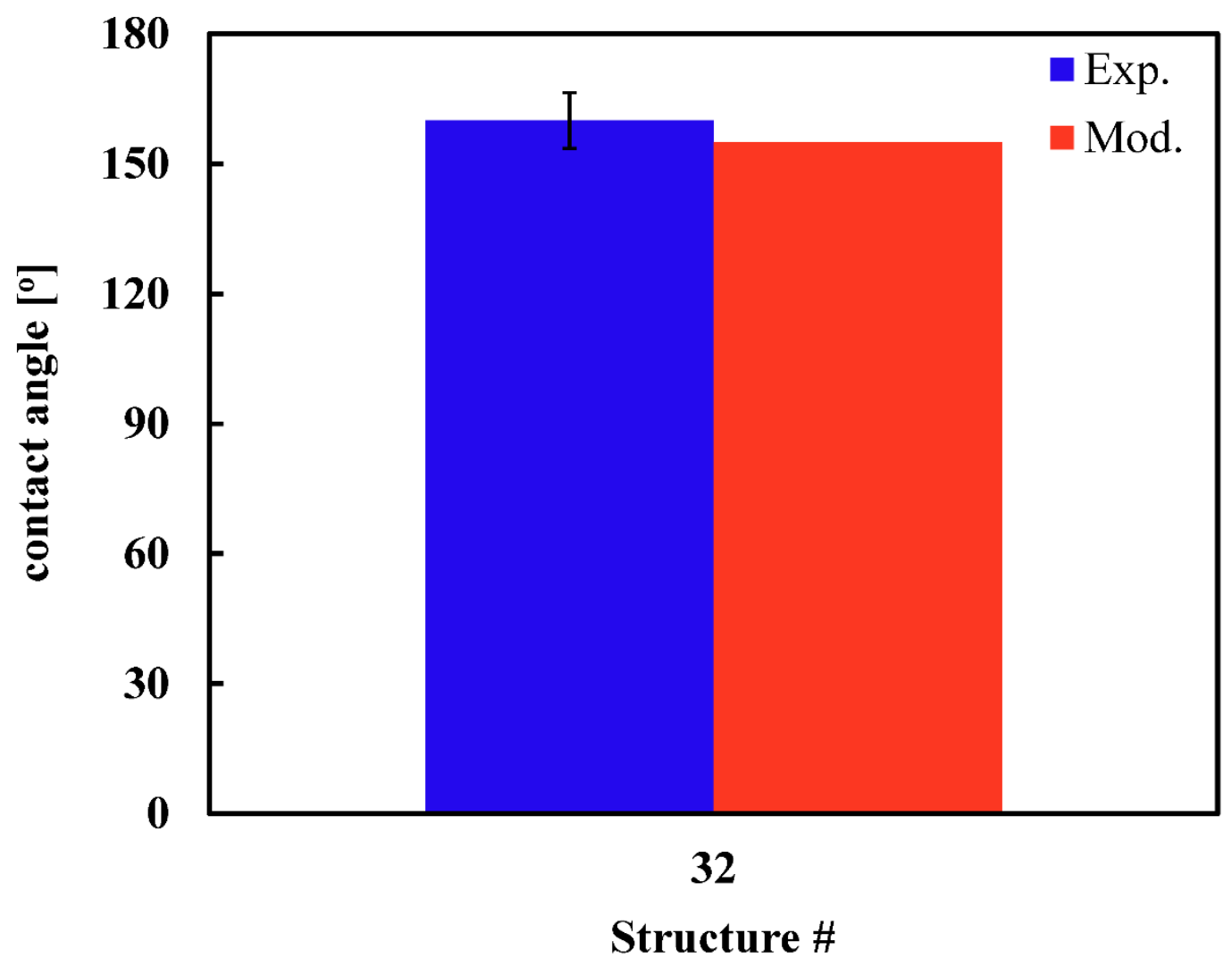

Figure 6. Contact angle comparison between model results (red) and experimental data (blue) for a three-level surface topography corresponding to three levels of sinusoids placed on top of each other (see Table 2).

\section{Conclusions}

The objective of this work is twofold; on one side, we report the extension of the formalism of our recently published wetting model [67] to account for 3D pillar topographies, while, on the other hand, we provide compelling evidence on the validation of our model in capturing accurately the wetting behavior across various topography types, surface materials and hierarchy levels. Realistic engineered surfaces featuring single-, two- and three-level surface topographies, found either in the literature or fabricated and analyzed in the context of this work, were utilized for validation purposes. One-to-one comparison between modeling results and experimental data validated our original theoretical hypothesis that, in general, multilevel hierarchical topographies enable enhanced liquid-repellency, constituting thereby a valuable tool for achieving superhydrophobicity. 
Of the four single-level surface topographies considered here, the 3D pillars exhibited the highest contact angles, in some cases exceeding $150^{\circ}$, owed to increased surface-to-volume ratio compared to the commensurate semi-infinite $2 \mathrm{D}$ textures. Subsequently, the fiber topography, presenting re-entrant geometry, yielded the largest contact angles among the single level 2D topographies, followed by the 2D pillars and sinusoidal topographies. Increased water repellency was, in general, observed for two-level hierarchical surfaces, while three-level sinusoidal hierarchical surfaces yielded contact angles as high as $165^{\circ}$. For the three-level sinusoidal topography, addition of a second level of sinusoidal patterns almost doubled the observed contact angle, while incorporation of a third sinusoidal level brought about an additional $12.5 \%$ contact angle increase. In all cases of multilevel topographies considered here, the different superposing levels had length scales differing in size from one another by at least one order of magnitude.

Our model predictions were for all single-level topographies considered here in gratifying agreement with experimental contact angle measurements resulting to an average error of 4.6\%, well within the experimental statistical uncertainty. Analogous comparisons for two- and three-level topographies resulted in average deviations of about 3.5\%. Taken all together, our refined wetting model captured remarkably well and within experimental inaccuracies the wetting behavior of water on various materials exhibiting single- and multilevel hierarchical roughness. Modelling wettability accurately across different wetting liquids, surface materials and topographies sets the foundations of a generic surface design methodology enabling tailored wetting properties for numerous technological applications relevant to packaging, circular economy of materials, waste reduction, recycling, as well as conservation of energy and natural resources.

\section{Acknowledgements}

NL and RK gratefully acknowledge the University of Applied Sciences Western Switzerland (HES-SO), the College of Engineering and Architecture Fribourg (HEIA-FR) and the MARKETPLACE (Materials Modelling Marketplace for Increased Industrial Innovation) project funded by the Horizon 2020 EU program under the NMBP-25-2017 call, with Grant agreement number 760173 (https:/the-marketplace-project.eu/) for financial support. PMK gratefully acknowledges the Swiss Nanoscience Institute (SNI) for funding of the projects PATCELL and REPALL with grant numbers 09.10 and 10.07 , respectively, under the 
framework of the Nanoargovia program. RDA acknowledges funding from the BIOSMART project belonging to the Bio Based Industries Joint Undertaking under the European Union's Horizon 2020 - Research and Innovation Framework Program with Grant agreement number 745762. Computational time and resources for this work were granted by the Department of Computer Science and Communication Systems at HEIA-FR.

\section{Data availability statement}

The data supporting the findings of this study is available from the corresponding author upon reasonable request.

\section{ORCID iDs}

Nikolaos Lempesis: $\quad$ https://orcid.org/0000-0002-4104-9666, Ruth Díez-Ahedo https://orcid.org/0000-0002-8033-0792, Per Magnus Kristiansen: https://orcid.org/0000-00017714-0966

\section{References}

[1] Adhikari B, Howes T, Bhandari B R and Truong V 2001 STICKINESS IN FOODS: A REVIEW OF MECHANISMS AND TEST METHODS International Journal of Food Properties 4 1-33

[2] Harte B R, Gray J I and Miltz J 1987 Food Product-Package Compatibility: Taylor \& Francis)

[3] Michalski M C, Desobry S and Hardy J 1997 Food materials adhesion: a review Critical reviews in food science and nutrition 37 591-619

[4] Criado M, Suárez B and Ferreirós C 1994 The importance of bacterial adhesion in the dairy industry Food Technology 48 123-6

[5] Nosonovsky M and Bhushan B 2008 Roughness-induced superhydrophobicity: a way to design non-adhesive surfaces Journal of Physics: Condensed Matter 20225009

[6] Höcker H 2002 Plasma treatment of textile fibers Pure and applied chemistry 74 423-7

[7] Zimmermann J, Reifler F A, Fortunato G, Gerhardt L-C and Seeger S 2008 A Simple, One-Step Approach to Durable and Robust Superhydrophobic Textiles Advanced Functional Materials 18 3662-9

[8] Shabanian S, Khatir B, Nisar A and Golovin K 2020 Rational design of perfluorocarbon-free oleophobic textiles Nature Sustainability

[9] Gao X, Yan X, Yao X, Xu L, Zhang K, Zhang J, Yang B and Jiang L 2007 The Dry-Style Antifogging Properties of Mosquito Compound Eyes and Artificial Analogues Prepared by Soft Lithography Advanced Materials 19 2213-7

[10] Cao L, Jones A K, Sikka V K, Wu J and Gao D 2009 Anti-Icing Superhydrophobic Coatings Langmuir 25 12444-8 
[11] Pan Q and Wang M 2009 Miniature Boats with Striking Loading Capacity Fabricated from Superhydrophobic Copper Meshes ACS Applied Materials \& Interfaces 1 420-3

[12] Ou J, Perot B and Rothstein J P 2004 Laminar drag reduction in microchannels using ultrahydrophobic surfaces Physics of Fluids 16 4635-43

[13] Marmur A 2006 Underwater Superhydrophobicity: Theoretical Feasibility Langmuir 22 14002

[14] Gurera D and Bhushan B 2019 Optimization of bioinspired conical surfaces for water collection from fog Journal of Colloid and Interface Science 551 26-38

[15] Wilson S J and Hutley M C 1982 The optical properties of'moth eye'antireflection surfaces Optica Acta: International Journal of Optics 29 993-1009

[16] Carlo M and Metin S 2006 A biomimetic climbing robot based on the gecko Journal of Bionic Engineering 3 115-25

[17] Arzt E, Gorb S and Spolenak R 2003 From micro to nano contacts in biological attachment devices Proceedings of the National Academy of Sciences 10010603

[18] Solga A, Cerman Z, Striffler B F, Spaeth M and Barthlott W 2007 The dream of staying clean: Lotus and biomimetic surfaces Bioinspiration \& biomimetics 2 S126

[19] Dean B and Bhushan B 2010 Shark-skin surfaces for fluid-drag reduction in turbulent flow: a review Philosophical Transactions of the Royal Society A: Mathematical, Physical and Engineering Sciences 368 4775-806

[20] Bushnell D M and Moore K J 1991 Drag reduction in nature Annual review of fluid mechanics 23 65-79

[21] Nosonovsky M and Bhushan B 2009 Superhydrophobic surfaces and emerging applications: Non-adhesion, energy, green engineering Current Opinion in Colloid \& Interface Science 14 270-80

[22] Nakajima A, Fujishima A, Hashimoto K and Watanabe T 1999 Preparation of Transparent Superhydrophobic Boehmite and Silica Films by Sublimation of Aluminum Acetylacetonate Advanced Materials 11 1365-8

[23] Chen W, Fadeev A Y, Hsieh M C, Öner D, Youngblood J and McCarthy T J 1999 Ultrahydrophobic and Ultralyophobic Surfaces: Some Comments and Examples Langmuir 15 3395-9

[24] Kijlstra J, Reihs K and Klamt A 2002 Roughness and topology of ultra-hydrophobic surfaces Colloids and Surfaces A: Physicochemical and Engineering Aspects 206 521-9

[25] Herminghaus S 2000 Roughness-induced non-wetting EPL (Europhysics Letters) 52165

[26] Sun T, Wang G, Feng L, Liu B, Ma Y, Jiang L and Zhu D 2004 Reversible switching between superhydrophilicity and superhydrophobicity Angewandte Chemie International Edition 43 357-60

[27] Zhang J, Lu X, Huang W and Han Y 2005 Reversible Superhydrophobicity to Superhydrophilicity Transition by Extending and Unloading an Elastic Polyamide Film Macromolecular Rapid Communications 26 477-80

[28] Lu X, Zhang C and Han Y 2004 Low-Density Polyethylene Superhydrophobic Surface by Control of Its Crystallization Behavior Macromolecular Rapid Communications 25 1606-10

[29] Seemann R, Brinkmann M, Herminghaus S, Khare K, Law B M, McBride S, Kostourou K, Gurevich E, Bommer S and Herrmann C 2011 Wetting morphologies and their transitions in grooved substrates Journal of Physics: Condensed Matter 23184108

[30] Khare K, Brinkmann M, Law B M, Herminghaus S and Seemann R 2009 Switching wetting morphologies in triangular grooves The European Physical Journal Special Topics 166 151-4

[31] Nishino T, Meguro M, Nakamae K, Matsushita M and Ueda Y 1999 The Lowest Surface Free Energy Based on -CF3 Alignment Langmuir 15 4321-3

[32] Tuteja A, Choi W, Ma M, Mabry J M, Mazzella S A, Rutledge G C, McKinley G H and Cohen R E 2007 Designing superoleophobic surfaces Science 318 1618-22 
[33] Chauhan P, Kumar A and Bhushan B 2019 Self-cleaning, stain-resistant and anti-bacterial superhydrophobic cotton fabric prepared by simple immersion technique Journal of Colloid and Interface Science $\mathbf{5 3 5}$ 66-74

[34] Li F, Bhushan B, Pan Y and Zhao X 2019 Bioinspired superoleophobic/superhydrophilic functionalized cotton for efficient separation of immiscible oil-water mixtures and oil-water emulsions Journal of Colloid and Interface Science 548 123-30

[35] Nanda D, Sahoo A, Kumar A and Bhushan B 2019 Facile approach to develop durable and reusable superhydrophobic/superoleophilic coatings for steel mesh surfaces Journal of Colloid and Interface Science 535 50-7

[36] Gurera D and Bhushan B 2018 Fabrication of bioinspired superliquiphobic synthetic leather with self-cleaning and low adhesion Colloids and Surfaces A: Physicochemical and Engineering Aspects 545 130-7

[37] Alinezhadfar M, Nasiri Khalil Abad S and Mozammel M 2020 Multifunctional cobalt coating with exceptional amphiphobic properties: self-cleaning and corrosion inhibition Surfaces and Interfaces 21100744

[38] Nasiri Khalil Abad S, Najibi Ilkhechi N, Adel M and Mozammel M 2020 Hierarchical architecture of a superhydrophobic Cd-Si co-doped TiO2 thin film Applied Surface Science 533147495

[39] Nasiri Khalil Abad S, Mozammel M, Moghaddam J, Mostafaei A and Chmielus M 2020 Highly porous, flexible and robust cellulose acetate/Au/ZnO as a hybrid photocatalyst Applied Surface Science 526146237

[40] Bhushan B and Martin S 2018 Substrate-independent superliquiphobic coatings for water, oil, and surfactant repellency: An overview Journal of Colloid and Interface Science 526 90-105

[41] Tuteja A, Choi W, Mabry J M, McKinley G H and Cohen R E 2008 Robust omniphobic surfaces Proceedings of the National Academy of Sciences 10518200

[42] Cheng Y-T and Rodak D E 2005 Is the lotus leaf superhydrophobic? Applied Physics Letters 86 144101

[43] Marmur A 2003 Wetting on Hydrophobic Rough Surfaces: To Be Heterogeneous or Not To Be? Langmuir 19 8343-8

[44] Nosonovsky M 2007 Multiscale Roughness and Stability of Superhydrophobic Biomimetic Interfaces Langmuir 23 3157-61

[45] Bittoun E and Marmur A 2012 The role of multiscale roughness in the lotus effect: is it essential for super-hydrophobicity? Langmuir 28 13933-42

[46] Nosonovsky M and Bhushan B 2008 Biologically inspired surfaces: broadening the scope of roughness Advanced Functional Materials 18 843-55

[47] Zhai L, Cebeci F C, Cohen R E and Rubner M F 2004 Stable superhydrophobic coatings from polyelectrolyte multilayers Nano letters 4 1349-53

[48] Chhatre S S, Choi W and Tuteja A 2010 Scale Dependence of Omniphobic Mesh Surfaces Langmuir 26 4027-35

[49] Ma M and Hill R M 2006 Superhydrophobic surfaces Current opinion in colloid \& interface science 11 193-202

[50] Barbieri L, Wagner E and Hoffmann P 2007 Water wetting transition parameters of perfluorinated substrates with periodically distributed flat-top microscale obstacles Langmuir 23 1723-34

[51] Kang S M, You I, Cho W K, Shon H K, Lee T G, Choi I S, Karp J M and Lee H 2010 One-step modification of superhydrophobic surfaces by a mussel-inspired polymer coating Angewandte Chemie International Edition 49 9401-4

[52] Young T 1805 III. An essay on the cohesion of fluids Philosophical Transactions of the Royal Society of London 95 65-87

[53] Wenzel R N 1936 Resistance of solid surfaces to wetting by water Industrial \& Engineering Chemistry 28 988-94 
[54] Cassie A B D and Baxter S 1944 Wettability of porous surfaces Transactions of the Faraday society 40 546-51

[55] Afferrante L and Carbone G 2010 Microstructured superhydrorepellent surfaces: effect of drop pressure on fakir-state stability and apparent contact angles Journal of Physics: Condensed Matter 22325107

[56] Afferrante L and Carbone G 2014 The effect of drop volume and micropillar shape on the apparent contact angle of ordered microstructured surfaces Soft Matter 10 3906-14

[57] Afferrante L and Carbone G 2015 Statistical theory of wetting of liquid drops on superhydrophobic randomly rough surfaces Physical Review E 92042407

[58] Ciavarella M and Afferrante L 2016 Adhesion of rigid rough contacts with bounded distribution of heights Tribology International 100 18-23

[59] Jung M, Brinkmann M, Seemann R, Hiller T, Sanchez de La Lama M and Herminghaus S 2016 Wettability controls slow immiscible displacement through local interfacial instabilities Physical Review Fluids 1074202

[60] Violano $G$ and Afferrante L 2019 Contact of rough surfaces: Modeling adhesion in advanced multiasperity models Proceedings of the Institution of Mechanical Engineers, Part J: Journal of Engineering Tribology 233 1585-93

[61] Marmur A and Bittoun E 2009 When Wenzel and Cassie Are Right: Reconciling Local and Global Considerations Langmuir 25 1277-81

[62] Bittoun E and Marmur A 2010 Chemical Nano-Heterogeneities Detection by Contact Angle Hysteresis: Theoretical Feasibility Langmuir 26 15933-7

[63] Carbone $G$ and Mangialardi L 2005 Hydrophobic properties of a wavy rough substrate The European Physical Journal E 16 67-76

[64] Bottiglione F and Carbone G 2013 Role of Statistical Properties of Randomly Rough Surfaces in Controlling Superhydrophobicity Langmuir 29 599-609

[65] Brown P S and Bhushan B 2016 Designing bioinspired superoleophobic surfaces APL Materials 4015703

[66] Brown P S and Bhushan B 2016 Durable, superoleophobic polymer-nanoparticle composite surfaces with re-entrant geometry via solvent-induced phase transformation Scientific reports 621048

[67] Lempesis N, Janka A, Gnatiuk O, van Eijndhoven S J L and Koopmans R J 2020 Predicting bioinspired candidate surfaces with superomniphobic characteristics Surface Topography: Metrology and Properties

[68] Vázquez U O M, Shinoda W, Moore P B, Chiu C-c and Nielsen S O 2009 Calculating the surface tension between a flat solid and a liquid: a theoretical and computer simulation study of three topologically different methods Journal of Mathematical Chemistry 45 161-74

[69] Zisman W A 1964 Contact Angle, Wettability, and Adhesion: AMERICAN CHEMICAL SOCIETY) pp 1-51

[70] Owens D K and Wendt R C 1969 Estimation of the surface free energy of polymers Journal of Applied Polymer Science 13 1741-7

[71] Ayyoob M and Kim Y J 2018 Effect of Chemical Composition Variant and Oxygen Plasma Treatments on the Wettability of PLGA Thin Films, Synthesized by Direct Copolycondensation Polymers (Basel) 101132

[72] Cheng C T, Zhang $G$ and To S 2016 Wetting characteristics of bare micro-patterned cyclic olefin copolymer surfaces fabricated by ultra-precision raster milling RSC Advances 6 1562-70

[73] Lv T, Cheng Z, Zhang D, Zhang E, Zhao Q, Liu Y and Jiang L 2016 Superhydrophobic Surface With Shape Memory Micro/Nanostructure and Its Application in Rewritable Chip for Droplet Storage ACS Nano 10 9379-86

[74] Lee S G, Lee D Y, Lim H S, Lee D H, Lee S and Cho K 2010 Switchable Transparency and Wetting of Elastomeric Smart Windows Advanced Materials 22 5013-7 
[75] Lin G, Zhang Q, Lv C, Tang Y and Yin J 2018 Small degree of anisotropic wetting on self-similar hierarchical wrinkled surfaces Soft Matter 14 1517-29

[76] Lee W-K, Jung W-B, Nagel S R and Odom T W 2016 Stretchable Superhydrophobicity from Monolithic, Three-Dimensional Hierarchical Wrinkles Nano Letters 16 3774-9 